CLINICOPATHOLOGICAL CONFERENCE

\title{
Fever in the recently returned HIV infected traveller
}

\author{
A Birnie, B G Gazzard, C Bacon, A Dogan, P J Shaw, R F Miller
}

Sex Transm Infect 2004;80:86-90. doi: 10.1136/sti.2003.008755

A 41 year old HIV infected man with an extensive travel history developed intermittent fever and weight loss shortly after returning from abroad. Extensive and prolonged investigation identified the cause.

See end of article for authors' affiliations

Correspondence to: Dr R F Miller, Centre for Sexual Health and HIV Research, Department of Primary Care and Population Sciences, Royal Free and University College Medical School, University College London, Mortimer Market Centre, Mortimer Market, off Capper Street, London WC1E 6AU, UK; Email: rmiller@gum.ucl.ac.uk

Accepted for publication 9 December 2003

\section{CASE PRESENTATION \\ (Dr A Birnie and Dr R F Miller)}

A 41 year old homosexual white man presented to the outpatient clinic. He reported $6 \mathrm{~kg}$ weight loss over the previous 5 months, together with intermittent fever, which had been present for the past month. The pattern of the fever was such that it lasted for 4 days, during which time he experienced-in addition-headache, malaise, and lethargy, and then he was free of symptoms for 10 days, until the cycle repeated itself. He had returned from Uganda 3 months before his clinic presentation.

The patient was found to be HIV-1 antibody positive in 1996: at the time of diagnosis his CD4 count was 480 cells $\times 10^{6} / 1$ and the HIV "viral load" was 11000 copies/ml. In 1997 while in Belize the patient received an insect bite on the leg. An eschar developed: biopsy of this was nondiagnostic. The clinical diagnosis was one of cutaneous leishmaniasis and the lesion resolved spontaneously over several weeks. In 2000 the patient had Shigella dysentery. In April 2002 he had presented to the outpatient clinic with a 1 year history of perianal discharge and intermittent diarrhoea. Investigations carried out between April and July 2002 (when the patient travelled to Uganda) included an endo-anal ultrasound, which showed an intersphincteric fistula; colonoscopy, which showed macroscopically normal mucosa; and colonic biopsies, which showed non-specific colitis; and analysis of multiple stool samples by microscopy for ova, cysts, and parasites and by culture for bacteria, protozoa, and fungi, which were negative. Other investigations included a full blood count which revealed haemoglobin $(\mathrm{Hb}) 12.5 \mathrm{~g} / \mathrm{dl}$, a normal white blood cell count (WBC), platelet count and blood film, normal urea and electrolytes, liver function and thyroid function tests, and $\mathrm{C}$ reactive protein (CRP). HBsAg and anti-HB core were not detected: anti-HBs was positive $(>100 \mathrm{mIU} / \mathrm{ml})$. Anti-HCV was not detected and an autoantibody screen, RA latex, ANA, ANCA, and ENA were all negative. The CD4 count was $>500$ cells $\times 10^{6} /$.

The patient was an occasional drinker $(<5$ units/week) and was a non-smoker. He had travelled extensively in the past 10 years-to
South America (Chile, Peru, Belize, Uruguay, Brazil, and Venezuela), North America (both east and west coasts), Africa (Botswana, Kenya, Uganda, South Africa), Asia (Thailand), Australia, New Zealand, and Europe (Scandinavia and Russia, as well as Spain and Portugal).

In mid-October 2002 the patient became rather more unwell and so was admitted to hospital. Examination at that time revealed widespread lymphadenopathy, more marked in the right than the left axilla and inguinal regions, the nodes being less than $1 \mathrm{~cm}$ in diameter and "firm" in consistency. In addition, $2 \mathrm{~cm}$ firm, smooth, non-tender hepatomegaly and $4 \mathrm{~cm}$ splenomegaly were noted. The skin was normal, apart from a fungal foot infection. Examination of the mouth, fundi, chest, central nervous system, and genitalia was normal.

Investigations at this stage revealed $\mathrm{Hb}=9.8 \mathrm{~g} / \mathrm{dl}, \mathrm{WBC}$, and platelet counts were normal; a blood film showed only a normocytic anaemia. Multiple blood films were negative for malaria. Biochemistry for urea and electrolytes, liver function, and resting venous lactate was normal. A lactate dehydrogenase enzyme level was $524 \quad($ normal $=240-480) \quad$ U/l. Serological tests for schistosomiasis, syphilis, Lyme disease, and leishmania (both DAT and K39) were all negative. Serology for cytomegalovirus (CMV) was IgG positive and IgM negative, indicating an old infection. In peripheral blood CMV was detected, by the polymerase chain reaction (PCR), at $<20$ copies/ml. Culture of multiple samples of blood, urine, and stool-for bacteria, mycobacteria, and fungi-was negative. A chest radiograph was normal. An abdominal ultrasound scan confirmed the clinical findings of hepatosplenomegaly; there was no intra-abdominal lymphadenopathy. A trans-thoracic echocardiogram was normal. The CD4 count was 290 cells $\times 10^{6} / 1$ and the HIV viral load was 23500 copies $/ \mathrm{ml}$.

What are your initial thoughts about this presentation? Are there any further investigations that you would request?

\section{DISCUSSION}

(Professor B G Gazzard)

This is a widely travelled HIV positive man. He has potentially been exposed to a wide variety of pathogens during his travels. Many of these, in the context of HIV induced immunosuppression, could cause this presentation. The normal appearance at colonoscopy with non-specific colitis on histology of colonic biopsies is almost universal in HIV infected individuals, so I don't think that this is an important finding. An initial thought is that in any HIV positive patient, particularly one with a relatively well preserved 
CD4 count who has an intermittent fever, lymphoma should be excluded. In the more immunosuppressed HIV infected patient non-Hodgkin's lymphoma should be excluded. In this patient with a normal CD4 count Hodgkin's disease should be considered in the differential diagnosis.

I think it is important to do a liver biopsy—as this organ is enlarged. If you were worried about the morbidity of this diagnostic procedure-then I'd go for a lymph node biopsy. Preferably this should be an axillary node-as inguinal nodes have a relatively lower diagnostic yield.

\section{CASE PRESENTATION}

\section{(Dr Birnie and Dr Miller)}

A right inguinal lymph node was biopsied. Histology showed only HIV "reactive" changes, and so a bone marrow aspirate and trephine were performed. This had a normal histological appearance. Special stains (Ziehl-Neelsen, periodic acid Schiff (PAS), Grocott, and Geimsa) and PCR for leishmania were negative on both the lymph node and bone marrow samples. The patient was discharged on 15 October 2002, with arrangements to attend the outpatient clinic for the results of the (above) investigations. The foot infection was treated with terbinafine.

The patient was readmitted to hospital on 30 October 2002, complaining of a 5 day history of productive cough, nasal congestion, and fever. On examination he was pyrexial, temperature $39^{\circ} \mathrm{C}$; in the chest there were widespread coarse crackles. The previously noted findings of lymphadenopathy and hepatosplenomegaly were unchanged. Investigations showed $\mathrm{Hb} 8.5 \mathrm{~g} / \mathrm{dl}$, WBC 1.6 (neutrophils 0.5 ) $\times 10^{9} / \mathrm{l}$, platelet count normal, CRP 230 (normal $<5.0 \mathrm{U} / \mathrm{ml}$ ). A chest radiograph showed patchy non-specific shadowing and a sinus radiograph showed maxillary sinus disease. Culture of blood, sputum, and urine for bacteria, mycobacteria, and fungi was negative. A bone marrow aspirate and biopsy were repeated.

What do you think that the repeat bone marrow examination will reveal?

\section{DISCUSSION}

\section{(Professor Gazzard)}

I wonder if it will reveal an underlying pathology-perhaps an infection or a lymphoma?

\section{PATHOLOGY}

\section{(Dr C Bacon and Dr A Dogan)}

The bone marrow showed absent mature granulopoiesis, with plentiful early precursors. There was an increase in eosinophil numbers and dyserythropoiesis. These findings were interpreted as showing transient agranulopoiesis: a drug reaction was suggested by the presence of eosinophils.

\section{CASE PRESENTATION}

\section{(Dr Birnie and Dr Miller)}

The patient was thought to be suffering from drug induced neutropenia, sinusitis, and a lower respiratory tract infection. The terbinafine was stopped as this has been reported as inducing neutropenia, and tazocin and gentamicin were started on an "empirical" basis.

Further investigations included bronchoscopy with bronchoalveolar (BAL) lavage, which revealed no endobronchial abnormality and an inflammatory pattern in BAL fluid consisting of neutrophils and lymphocytes. Staining and culture of BAL fluid was negative for bacteria, mycobacteria, fungi, and parasites (including Leishmania spp); serology on BAL for RSV, parainfluenza $1-3$, and influenza a and b was negative. Multiple blood films were negative for malaria. Serology was negative for Toxoplasma, amoeba, filaria (Wucheria bancrofti antigen was also negative), Brucella (abortus and mellitensis), schistosomiasis, Trypanosoma (brucei and cruzei), Rickettsia (typhus and spotted fever), Bartonella spp, Leishmania (both DAT and K39), Cryptococcus (CrAg), syphilis (THPA), and hepatitis C. Acute serology for Coxiella burnetii was sent to the reference laboratory. Additional imaging was performed.

\section{IMAGING}

\section{(Dr P J Shaw)}

Computed tomography (CT) scanning of the head showed only maxillary sinus disease. CT of the chest showed multilobar consolidation, discrete nodules, small airways disease, and ground glass shadowing centred on the bronchovascular bundles. Small pleural and pericardial effusions were present. The intrathoracic findings have a wide infective differential diagnosis, including invasive aspergillosis (given the history of neutropenia). Lymphoma can also give a similar appearance. CT of the abdomen showed hepatosplenomegaly, minimal ascites, and widespread small volume $(1-2 \mathrm{~cm})$ intra-abdominal lymphadenopathy (fig l) compatible with either an infective process (for example, mycobacterial infection) or with lymphoma.

\section{CASE PRESENTATION}

\section{(Dr Birnie and Dr Miller)}

What further investigations would you want to carry out at this stage?

\section{DISCUSSION}

(Professor Gazzard)

I still want to know what the liver biopsy shows.

\section{PATHOLOGY}

\section{(Dr Bacon and Dr Dogan)}

The liver biopsy showed haemosiderosis and sinusoidal dilatation, which are non-specific findings. Special stains (Ziehl-Neelsen, PAS, Grocott, and Giemsa) were negative. PCR for Leishmania was negative.

\section{CASE PRESENTATION}

\section{(Dr Birnie and Dr Miller)}

The patient made a steady recovery from the upper and lower respiratory tract symptoms over several days. His fever settled for 7 days and then recurred. A follow up CT scan of the thorax, performed after a 12 day interval showed a dramatic improvement in the lung parenchymal abnormalities, making lymphoma unlikely as a cause of the CT appearances. In

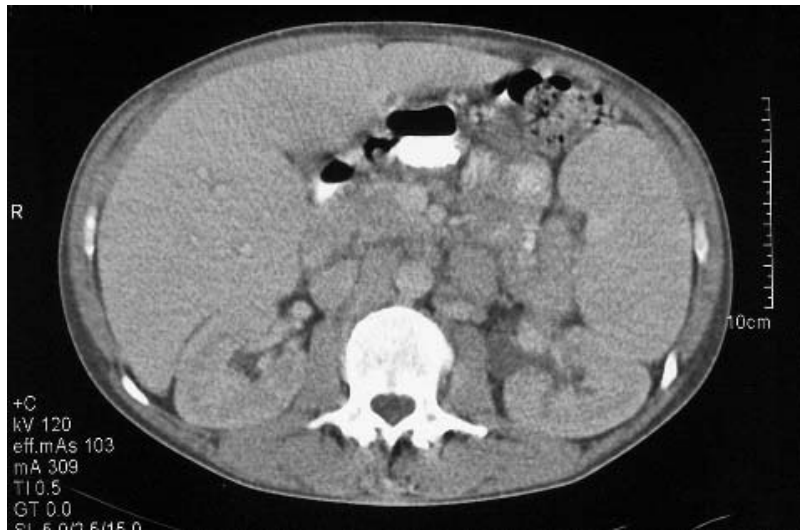

Figure $1 \mathrm{CT}$ of the abdomen showing diffuse hepatosplenomegaly. Multiple enlarged nodes are demonstrated in the porta and retroperitoneum, the largest in the left para-aortic region measuring $3 \times 2 \mathrm{~cm}$. 
blood inflammatory markers remained elevated, with CRP $>200 \mathrm{U} / \mathrm{l}$ and erythrocyte sedimentation rate (ESR) $210 \mathrm{~mm}$ in the first hour. Just before discharge from hospital in December 2002 the CD4 count was 210 cells $\times 10^{6} / 1$. The patient was offered HAART, but declined as he wanted to defer antiretroviral therapy for two reasons: firstly, to see if his CD4 count recovered following recovery from his intercurrent infection and, secondly, he wished to travel again. He did not start primary prophylaxis against Pneumocystis jiroveci pneumonia.

Would you like to comment on this scenario?

\section{DISCUSSION}

\section{(Professor Gazzard)}

This patient, with a falling CD4 count, has cyclical fever, lymphadenopathy, hepatosplenomegaly, and a drug induced episode of neutropenic sepsis. An infection screen is negative and there are non-diagnostic results from two bone marrow examinations, a liver biopsy and a lymph node. This could still be caused by an occult lymphoma, or to an infective process.

\section{CASE PRESENTATION \\ (Dr Birnie and Dr Miller)}

At this stage our differential diagnosis was rather wide! We wondered about the possibility of occult infection, despite the negative results. We thought that tuberculosis was unlikely, given the cyclical nature of the patient's fever. We also wondered about the possibility of an underlying malignancy, such as a lymphoma. Finally, we wondered if all of the patient's problems, before the drug induced neutropenic sepsis, might be ascribed to HIV "constitutional disease." In order to try to exclude the possibility of an underlying malignancy we carried out a whole body ${ }^{18}$ FDG positron emission tomography (PET) scan. The appearances were those frequently seen in patients with HIV infection'; there was no evidence of malignancy (fig 2).

The patient was seen in the outpatient department for follow up in January 2003, 2 weeks after discharge from hospital. At that time he reported persistent cyclical fever and malaise with tiredness. He had recovered from the episode of respiratory infection. At this time investigations showed $\mathrm{Hb}$ $6.9 \mathrm{~g} / \mathrm{dl}, \mathrm{WBC} 2.7 \times 10^{9} / \mathrm{l}$, and platelets $=189 \times 10^{9} / \mathrm{l}$. The DAT IgG was weakly positive and haptoglobins were normal. The CD4 count was 210 cells $\times 10^{6} / 1$ and HIV viral load was 50000 copies $/ \mathrm{ml}$. The patient was transfused with whole blood and at the end of January 2003 began HAART, with a combination of lamivudine, tenofovir, and efavirenz.

Do you have any comments on this regimen of HAART? We were trying to avoid using a combination that might interact with antimycobacterial drugs, if these became necessary, and we also wanted to give the patient a regimen that was a low pill burden—as he was intending to travel again.

\section{DISCUSSION}

\section{(Professor Gazzard)}

I'm not sure that ascribing this patient's fever to HIV constitutional disease is a good idea. Several studies of HIV infected patients with fever have shown that only rarely is HIV itself the cause of the fever. ${ }^{2-5}$ This is an unusual first line regimen of HAART. Any regimen containing efavirenz is likely to be very effective. I'm not sure that you need to worry about drug-drug interactions between HAART and antimycobacterial drugs, given the negative results from multiple cultures and in the light of your observation about the cyclical nature of the patient's fever, I don't think that this patient has mycobacterial infection. This particular featurethe cyclical fever-makes me wonder about a "Pel-Ebstein" fever. ${ }^{6} \mathrm{I}^{\prime} \mathrm{m}$ thinking of lymphoma once more.

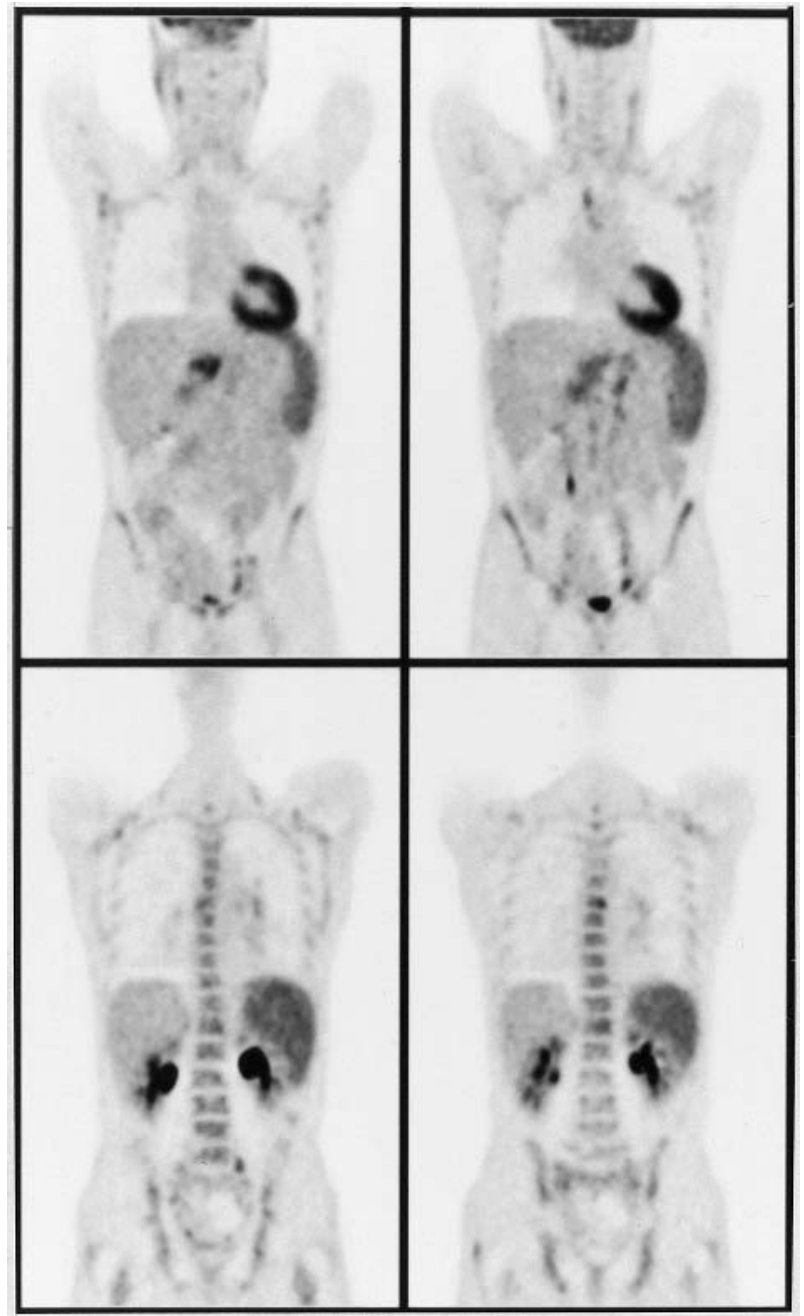

Figure 2 Whole body ${ }^{18} \mathrm{FDG}$ PET (coronal slice). There is avid uptake of ${ }^{18} \mathrm{FDG}$ in the temporal lobes and the left ventricle of the myocardium (these sites are very metabolically active and these appearances are normal). There is minor uptake in the axilllae, the cervical region, within the thorax (paratracheal, bronchial, and subcarinal nodes), and the abdomen (coeliac axis, mesenteric, iliac, and inguinal nodes). There is increased activity in the spleen - which is also slightly enlarged and slight increased uptake in the vertebrae. These are typical appearances in HIV infected patients. There are no features suggestive of malignancy.

\section{CASE PRESENTATION}

\section{(Dr Birnie and Dr Miller)}

The patient was adherent to his antiretroviral therapy and by mid-April 2003 he had gained $8 \mathrm{~kg}$ in weight, his CD4 count was 400 cells $\times 10^{6} / \mathrm{l}$, and HIV viral load was $<50$ copies $/ \mathrm{ml}$. His fever had lysed during the first few days of receiving HAART, but had subsequently returned, with the same cyclical pattern. A persistent anaemia was noted, $\mathrm{Hb} 8 \mathrm{~g} / \mathrm{dl}$, and there was a persistent acute phase response, with elevations of CRP and ESR.

The patient was readmitted to hospital on 23 April 2003 for an elective blood transfusion and for reassessment. On examination the findings of lymphadenopathy and hepatosplenomegaly were unchanged. At this point the results of acute and convalescent serology for $C$ burnetii became available from the reference laboratory. The results showed in the "acute" sample (obtained 31 October 2002) that phase I IgG was $1: 160$ and IgM was negative; phase II IgG was 1:160 and IgM was negative. In the "convalescent" sample phase I IgG was 1:160 and IgM was negative, phase II IgG was $1: 1280$ and $\operatorname{IgM}$ was negative. 

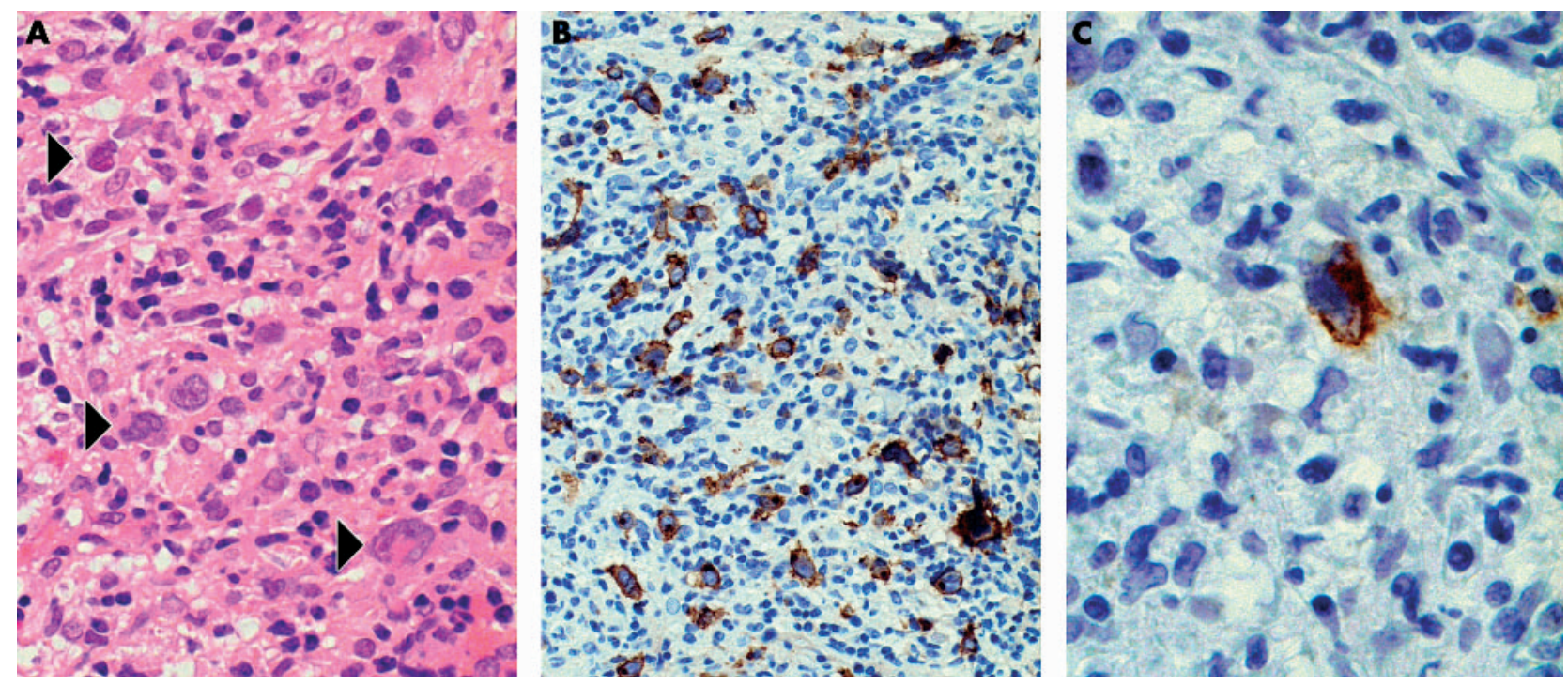

Figure 3 Bone marrow biopsy. (A) Haematoxylin and eosin (magnification $\times 400$ ) showing (arrows) both multinucleated Reed-Sternberg cells and mononuclear Hodgkin cells. (B) Immunoperoxidase: the neoplastic cells express CD30 (magnification $\times 400$ ). (C) Immunoperoxidase, a subset of neoplastic cells express EBV-LPM-1 (magnification $\times 800)$.

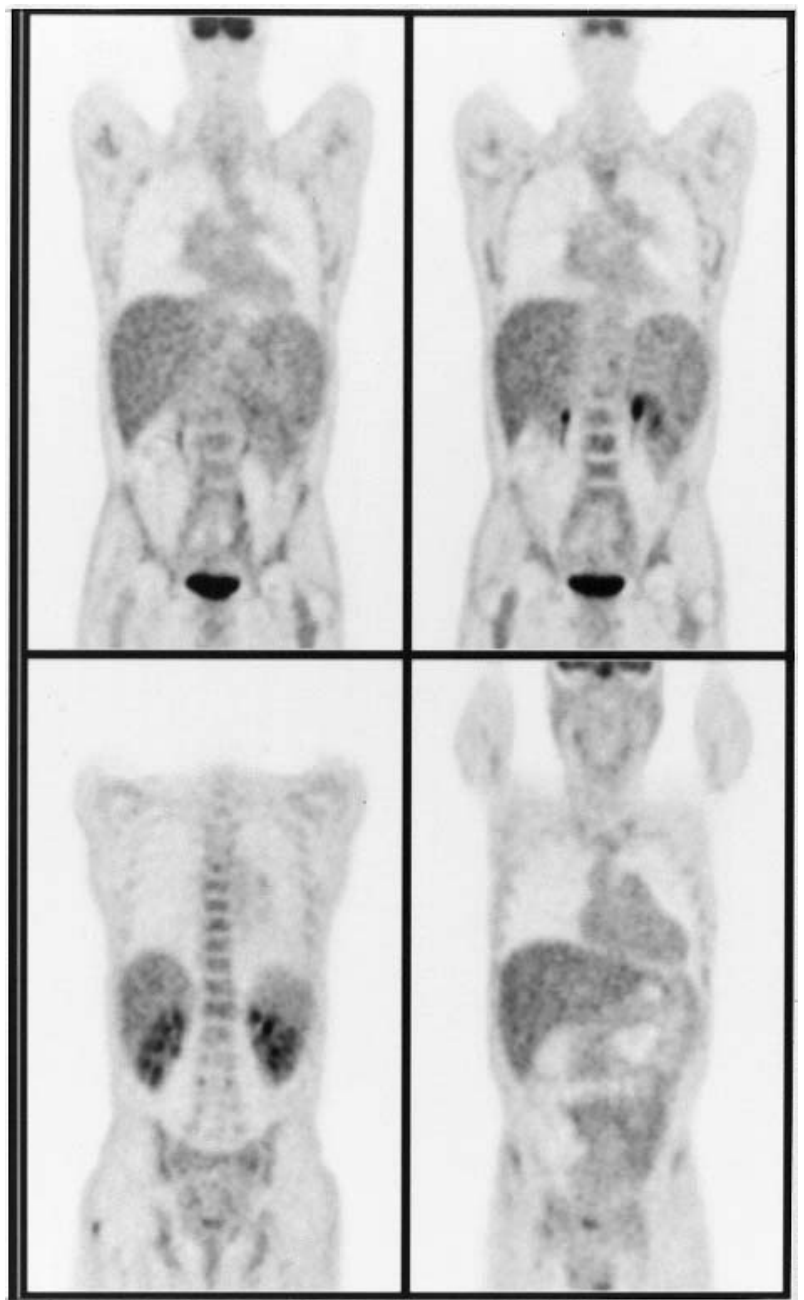

Figure 4 Whole body ${ }^{18}$ FDG PET scan (coronal slice). There is marked reduction in activity in most lymph node groups. Of note, activity is reduced in the spleen and in the vertebrae. These appearances show a response to HAART and no evidence of malignancy.
What is the significance of these results? Do they contribute to making a diagnosis in this patient?

\section{DISCUSSION \\ (Professor Gazzard)}

These serological data are compatible with acute Q feverthat is, an acute $C$ burnetii infection. In acute $Q$ fever the phase II IgG response is greater than the phase I IgG response. I don't think that this in any way explains this patient's original presentation. I think that the patient had an intercurrent acute $C$ burnetii infection with pneumonia in October 2002. ${ }^{7}$ There are no features in this serology to suggest that the patient has a chronic $C$ burnetii (" $\mathrm{Q}$ " fever) infection.

\section{CASE PRESENTATION}

\section{(Dr Birnie and Dr Miller)}

We were uncertain about interpretation of the $C$ burnetii serology in an immunosuppressed HIV infected patient and so began empirical treatment with doxycycline and chloroquine-the aim being to give 2 weeks' treatment. The patient did not tolerate this regimen, or a combination of doxycycline and ofloxacin. By early May 2003 the patient's fever had worsened: non-steroidal anti-inflammatory drugs and glucocorticoids (prednisolone, up to $60 \mathrm{mg}$ per day) were of no benefit. A further bone marrow aspirate and trephine were performed, in order to search for granulomata, in support of a possible diagnosis of chronic (granulomatous) Q fever.

Do you have any comments about this management plan? Do you want to suggest a diagnosis?

\section{DISCUSSION}

\section{(Professor Gazzard)}

I'm sure that this patient does not have chronic Q fever. However the repeat bone marrow investigation will be useful, as it may now reveal the diagnosis. I'm still thinking about the possibility of a disseminated infection or of Hodgkin's lymphoma. I am drawn back to an observation that was made early on in this patient's presentation-that there was elevation of the peripheral blood lactate dehydrogenase enzyme level. This, in the context of lymphoproliferative disease might suggest an underlying lymphoma; in the 
context of some infections-for example, histoplasmosis; this finding supports a diagnosis of disseminated infection. ${ }^{8} \mathrm{My}$ differential diagnosis remains either a disseminated infection-for example, histoplasmosis, or a lymphoma, and I'd favour Hodgkin's disease over a non-Hodgkin's lymphoma.

\section{PATHOLOGY}

\section{(Dr Bacon and Dr Dogan)}

The bone marrow was hypercellular with areas of disorganised trilineage, maturing haematopoiesis, and areas of fibrosis containing numerous large atypical cells with the morphology of Reed-Sternberg cells and Hodgkin cells in a background of inflammatory cells, including numerous eosinophils (fig 3A). Immunocytochemistry showed these cells were strongly positive for CD30 (fig 3B) and EpsteinBarr virus-latent membrane protein-1 (EBV-LMP-1) (fig 3C), but not for the B cell markers CD20 and CD79a and the $\mathrm{T}$ cell marker $\mathrm{CD} 3$. The appearances were considered to be diagnostic of classic Hodgkin's lymphoma involving the bone marrow.

\section{FINAL DIAGNOSIS}

(1) HIV infection

(2) Intercurrent acute Coxiella burnetii infection (Q fever)

(3) Hodgkin lymphoma-stage IV; presenting in bone marrow.

\section{CASE PRESENTATION}

\section{(Dr Birnie and Dr Miller)}

The patient underwent staging investigations, to determine the extent of the lymphoma. A CT scan of the thorax showed only axillary lymphadenopathy and a CT scan of the abdomen showed diffuse hepatosplenomegaly and intraabdominal lymphadenopathy, as previously described. A repeat ${ }^{18} \mathrm{FDG}$ PET scan showed that there was persistent minor uptake of tracer in lymph nodes (fig 4) and no evidence of uptake in the liver, spleen, intra-abdominal lymph nodes, or bone marrow. The appearances were compatible with those seen in an HIV infected patient who is receiving antiretroviral therapy and further suggested that the Hodgkin's lymphoma was limited to the bone marrow.

The patient continued with HAART and in June 2003 began combination chemotherapy, with ABVD (doxorubicin, bleomycin, vinblastine, and dicarbazine), as treatment of the Hodgkin's lymphoma. Six months later he was in complete remission and was asymptomatic. His CD4 count remained $>400$ cells $\times 10^{6} / 1$ and the HIV viral load was undetectable.

In HIV uninfected individuals Hodgkin's disease usually spreads locally-to contiguous lymph node groups; spread to extranodal tissue is a late occurrence. In patients with HIV infection who have Hodgkin's disease non-contiguous spread of lymphoma may be seen-for example, splenic involvement, without liver involvement. Bone marrow involvement is common ${ }^{9-12}$ - occurring in $40-50 \%$ of HIV infected patients with Hodgkin's disease. Between 74\% and 92\% of HIV infected patients with Hodgkin's disease have extensive disease (Ann Arbor stage III or IV) at presentation ${ }^{10}$; isolated bone marrow Hodgkin's disease is rare. ${ }^{13}$

\section{Key points}

- Fever in an HIV infected patient is rarely the result of the effects of HIV itself and identification of a specific cause should be actively pursued

- Relapsing or intermittent fever in a patient with HIV infection may suggest an underlying lymphoma as the cause

\section{ACKNOWLEDGEMENTS}

This case was presented at the Midlands branch of the Medical Society for the Study of Venereal Diseases on 19 September 2003. On that occasion the case was presented by Dr RF Miller and was discussed "blind" by Professor BG Gazzard. We thank Professor D Mabey, Professor P Chiodini, Dr SG Wright, and Dr T Doherty, all of the Hospital for Tropical Diseases, for advice on patient management.

\section{Authors' affiliations}

A Birnie, R F Miller, Patrick Manson Unit, University College London Hospitals NHS Trust, Centre for Sexual Health and HIV Research, Department of Primary Care and Population Sciences, Royal Free and University College Medical School, University College London and Camden PCT, Mortimer Market Centre, London WCIE 6 AU, UK

B G Gazzard, Department of HIV/GU Medicine, Kobler Centre, Chelsea and Westminster Hospitals NHS Trust, London SW10 9NH, UK

C Bacon, A Dogan, Department of Histopathology, Royal Free and University College London Medical School, University College London, London WC1E 6AU, UK

P J Shaw, Department of Imaging, University College London Hospitals NHS Trust, London WC1E 6AU, UK

\section{REFERENCES}

1 Scharko AM, Perlman SB, Pyzalski RW, et al. Whole-body positron emission tomography in patients with HIV-1 infection. Lancet 2003;362:959-61.

2 Sepkowitz KA, Telzak EE, Carrow M, et al. Fever among outpatients with advanced human immunodeficiency virus infection. Arch Intern Med 1993; 153:1909-12.

3 Miller RF, Hingorami AD, Foley NM. Pyrexia of undetermined origin in patients with human immunodeficiency virus infection and AIDS. Int J STD AIDS 1996:7:170-5.

4 Lambertucci JR, Rayes AA, Nunes F, et al. Fever of undetermined origin in patients with the acquired immunodeficiency syndrome in Brazil: report on 55 cases. Rev Inst MED Trop Sao Paulo 1999:41:27-32.

5 Armstrong WS, Katz JT, Kazaniian PH. Human immunodeficiency virusassociated fever of unknown origin: a study of 70 patients in the United States and review. Clin Infect Dis 1999;28:341-5.

6 Zuger A. Relapsing fever in an HIV-infected man. AIDS Clin Care 1995:7:56-9.

7 Marrie TJ. Coxiella burnetii pneumonia. Eur Respir J 2003;21:713-19.

8 Graviss EA, Vanden Heuvel EA, Lacke CE, et al. Clinical prediction model for differentiation of disseminated Histoplasma capsulatum and Mycobacterium avium complex infections in febrile patients with AIDS. J Acquir Immune Defic Syndr 2000:24:30-6.

9 Ree HJ, Strauchen JA, Khan AA, et al. Human immunodeficiency virusassociated Hodgkin's disease. Clinicopathologic studies of 24 cases and preponderance of mixed cellularity type characterized by the occurrence of fibrohistoid stromal cells. Cancer 1991;15:1614-21.

10 Vaccher E, Spina M, Tirelli U. Clinical aspects and management of Hodgkin's disease and other tumours in HIV-infected individuals. Eur J Cancer 2001;37:1306-15

11 Karcher DS. Clinically unsuspected Hodgkin disease presenting initially in the bone marrow of patients infected with the human immunodeficiency virus. Cancer 1993:71:1235-1238.

12 Calza L, Manfredi R, Colangeli V, et al. Hodgkin's disease in the setting of human immunodeficiency virus infection. Scand J Infect Dis 2003;35:136-41

13 Ponzoni M, Fumagalli L, Rossi G, et al. Isolated bone marrow manifestation of HIV-associated Hodgkin lymphoma. Mod Pathol 2002;15:1273-8. 